\title{
PER RECTAL MAGNETIC INFRA- RED LASER THERAPY (MILT) BY OPTICAL PROBE AND HAEMATOSPERMIA WITH CHRONIC PELVIC PAIN SYNDROME (CPPS) / CHRONIC PROSTATITIS
}

\author{
ABDUL MATIN ANAMUR RASHID CHOUDHURY¹, TASMINA PARVEEN²
}

\begin{abstract}
:
Objective : To observe improvement of symptoms of haematospermia after treatment with Magnetic infra-red laser therapy (MIL-therapy) in patients ofhaematospermia with Chronic Prostatitis.

Method: Observational study was done on 30 male patients with haematospermiawith CPPS or Chronic prostatitis, age range varied from 30 to 60 years. The study was conducted from 2004 to 2008 in a private medical center in Dhaka city. The MIL therapy in use has a wave length of $1000 \mathrm{~Hz}$. The Laser beam reaches the prostate and seminal vesical with a special optic probe. In this study the prostate anad seminal vesicle was examined per rectally by doing digital rectal examination with use of gloves and gel. The treatment schedule was one treatment every two days (treatment time of 5 to 10 minutes , wave length $1000 \mathrm{~Hz}$ ) for a total 10 application. After 10 application patients were asked for follow-up and data were collected and analysed .
\end{abstract}

Result :Among 30 patients of Haematospermia with Chronic prostatitis aged between 30-60 years with complains of blood discharge in semen were under treatment with MIL therapy. 25 patients with haematospermia were cured and 04 patients improved after laser treatment. But 01 patient found not improved on patients history.

Conclusion : Complains of patients and sign -symptoms cured and improved after MIL therapy.

Key words: MIL Therapy, Haematospermia, CPPS/Chronic prostatitis.

Bangladesh J. Urol. 2019; 22(2): 170-176

\section{Introduction :}

Laser means Light Amplification by Stimulated Emission of Radiation and was first theorized by Einstein.

Magnetic Infra -red laser therapy is a regulative medical treatment modality, which is used most in medical specialties including urological aspect[1].

Haematospermia means blood in semen (called haematospermia because sperm are mixed with blood in the semen) can be frightening symptoms but it is

1. Associate Professor, Department of Urology, National Inistitute of Kidney Diseases and Urology, Dhaka.

2. Professor, Department o Physiology, Uttara Adhunik Medical College, Uttara, Dhaka

Correspondence : A.M. Anamur Rashid Choudhury, Department of Urology, National Inistitute of Kidney Diseases and Urology, Dhaka. E-mail :dr_choudhury2001@yahoo.com

Received: 05 February 2019

Accepted: 10 May 2019 usually not a sign of serious. Blood in semen is not usually a sign of cancer. It does not affect sexual function. Semen is composed of sperm from the epididymis and fluid from the seminal vesicle,Prostate and small mucous gland that provide nutrition to sperm. Thus blood could come from injury to any of these structures [2,3].

Warning signs:Certain symptoms and characteristics are cause for concern. They include : 1)Bleeding lasting longer than 1 month (unless there was a recent prostate gland biopsy) 2) A lump that can be felt in the scrotum $3)$ Travel to a region where schistosomiasis is prevalent[4,5,6].

Physical Examination : When the man first noticed the blood :Biopsy of the prostate gland, Urinary tract infection, Bleeding disorder, Benign Prostatic 
Per Rectal MILT by optical probe and Haematospermia with Chronic pelvic pain syndrome (CPPS) /Chronic Prostatitis

Hyperplasia, Examination of Genitalia :By DRE — to exclude Prostate cancer ,Benign Prostatic Hyperplasia, Prostatitis.

Investigations done : Men younger than 35 to 40 years , and men who recently had a prostate biopsy. Blood in semen is not serious and resolve on its own .i)Urinalysis,ii)Urine culture iii)Test for Prostate cancer (men over 40 years ) :a) Serum PSA (Prostate -specific -Antigen) ,b)Transrectal ultrasonography (TRUS) c) MRI d) Cystoscopye) Semen inspection and analysis are rarely done.

Prostatitis is any form of inflammation of the prostate gland. Because women do not have a prostate gland, it is a condition only found in men, although women do have microscopic paraurethral Skene's glands connected to the distal third of the urethra in the prevaginal space that are homologous to the prostate, and may cause symptoms. ${ }^{6}$

In chronic prostatitis/chronic pelvic pain syndrome (CP) CPPS) there is Pelvic pain of unknown cause, lasting longer than 6 months, as the key symptom. Symptoms may wax and wane. Pain can range from mild discomfort to debilitating. Pain may radiate to back and rectum, making sitting difficult. Dysuria, arthralgia, myalgia, Unexplained fatigue, Abdominal pain, and frequency may all be present. Frequent urination and increased urgency may suggest interstitial cystitis (inflammation centred in bladder rather than prostate). Ejaculation may be painfulas the prostate contracts during emission of semen, although nerve- and musclemediated post-ejaculatory pain is more common, and a classic sign of CP/CPPS.Passage of blood with semen (Haematospermia) during ejaculation ..7,8,9

Laser therapy has analgesic effect due to measurable changes in the potential of nerve cell membranes lead to its hyperpolarization as a good analgesic mechanism. It has improved release reaction of endorphins and changes in transmitter concentration at the synapsis.

MILThas also Anti - Inflammatory effect due to Enhancement of proliferation of immune cells (enhancement of immune response, increase of NK cell activity etc.), Enhances lymphatic activity (drainage), Improves microcirculation (vasodilation), Reduces swelling (oedema\&haematoma resorption) and Increases ATP production.

\section{Theoretical Fundamentals of MIL-Therapy:}

Essential principles of MIL-Therapy:

Clinical effects: Activation of albumen (RNA, DNA) Synthesis, activation of ferments, increase of ATP output, improvement of blood circulation, content and functions, regeneration of tissues, reinforcement of collagen synthesis, anti-inflamatory effect, antiedematous effect, anaesthetisating effect, cholesterol level reduction, stimulation of specific and non-specific immunity factors, powerful antioxidant effect, prostaglandin synthesis raise and lipid peroxidal oxidizing level decrease (Fig. 1,2,3).

\section{Biological Principles of MIL-Therapy:}

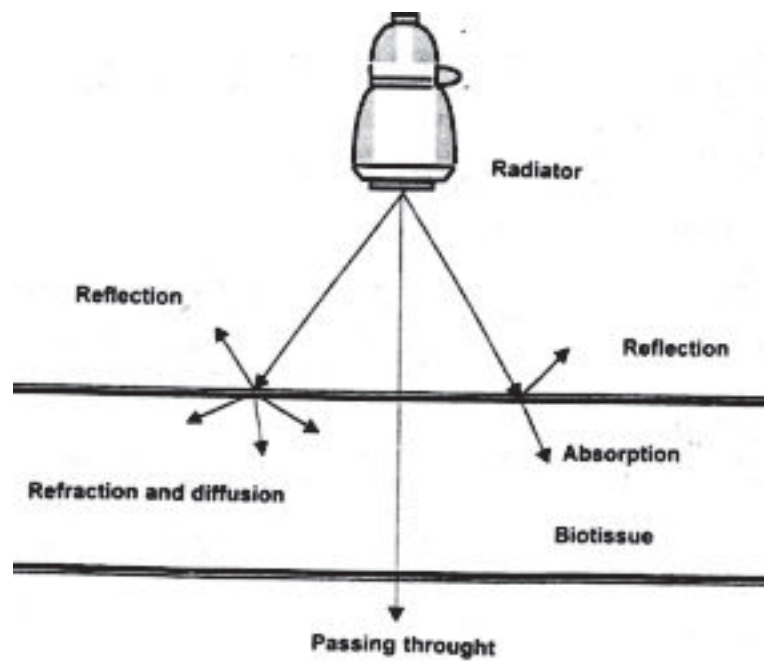

Fig.-1: Red irradiation passing through biotissue

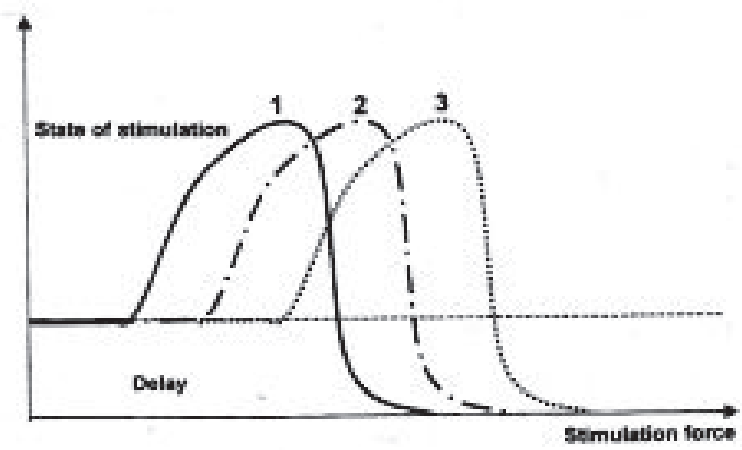

Fig.-2: Schematic interpretation of laser ray and biotissue

Over all pathophysiology of laser therapy in the correct

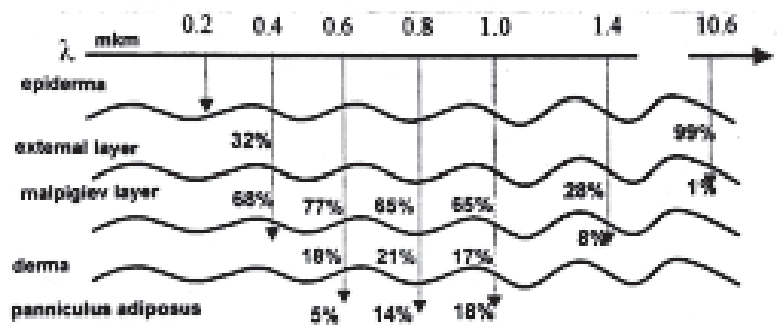

Fig.-3: Depth of laser irradiation penetration through biotissue 
Mechanism of action of MILT:

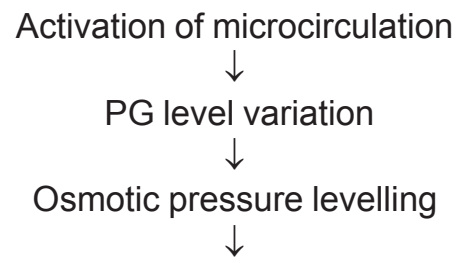

Tissue dropsicality decrease

\section{Reparative process stimulation}

Light quantum

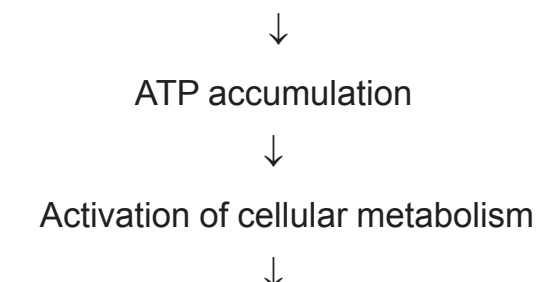

Synthesis of albumen and collagen

New capillary formation
Activation of neurone metabolism

$\downarrow$

Raise of endorphin level

$\downarrow$

Increase of pain sensitivity threashold

$\downarrow$

\author{
Stimulation of Immune response \\ Light quantum \\ $\downarrow$ \\ Proliferation of Immune cells \\ $\downarrow$ \\ Increase of IM globulins \\ $\downarrow$
}

Stimulation of physiological functions. frequency, the red light is absorbed within the mitochondria and the infrared light is absorbed at the cell membrane.In the cell of prostate gland this results in a change in membrane permeability, increased ATP levels and increased DNA production. So there is cellular hyperplasia of prostate and increased activity of ATP dependant $\mathrm{Na}-\mathrm{K}$ pump ${ }^{1,2,3}$.

\section{Materials and methods:}

This study was an observational study done on30 male patients with haematospermia age range varied from 30 to 60 years. The study period was from 2009 to 2011 in a private medical center at Dhaka city. The MIL-therapy in use has a wave length of $904 \mathrm{~nm}$ and a frequency of $3000 \mathrm{~Hz}$ (Fig. 4,5). The Laser beam reaches the prostate with a special optic probe. In this study the patient is examined per rectally after doing digital rectal examination with use of gloves and gel. It can be sterilized and it is atoxic. During examination blood found in the condom (Fig. 6). At the beginning we used a "Laser Super Sonic" machine with endorectal probe according to Strada. The treatment schedule was 1 treatment every two days (treatment's time of 5/10 minutes, wave length $1000 \mathrm{KHz}$ )) for a total of 10 applications. Transrectal laser therapy was not indicated in prostate larger than $4 \mathrm{~cm}$ because this is the maximum depth of the laser beam's efficacy.

Inclusion Criteria : Age -31 to $60 \mathrm{yrs}$, in DRI findings Prostate size normal, in USG findings Prostate size normal, Haematospermia present, Pelvic pain Present, Dysuria present, Sign symptoms of Chronic prostatitis present .

Exclusion criteria : Age $<31$ yrs and $>60$ Yrs. , No haematospermia, No Sign symptoms of Prostatitis , No pelvic pain , No dysuria .

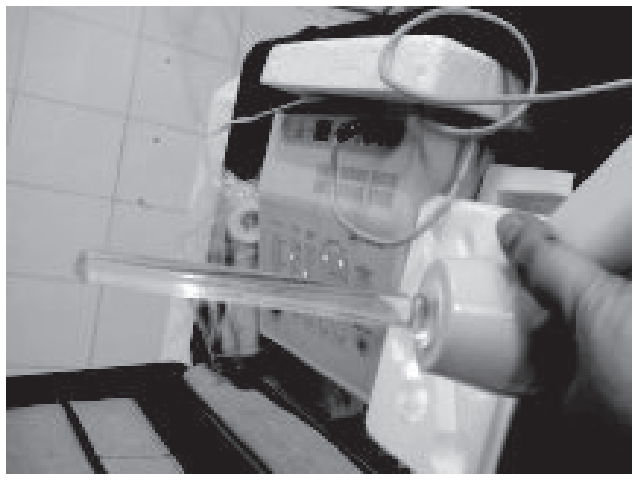

Fig.-4: Optical probe 
Per Rectal MILT by optical probe and Haematosperrmia with Chronic pelvic pain syndrome (CPPS) /Chronic Prostatitis

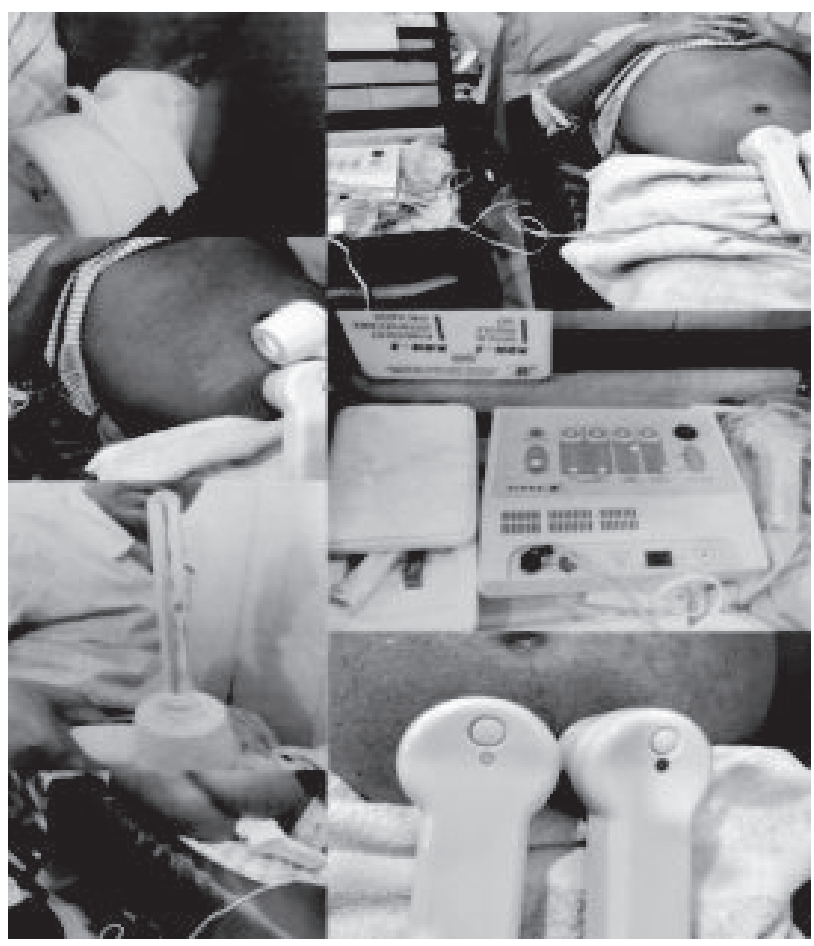

Fig.-5: Procedure of MIL Therapy

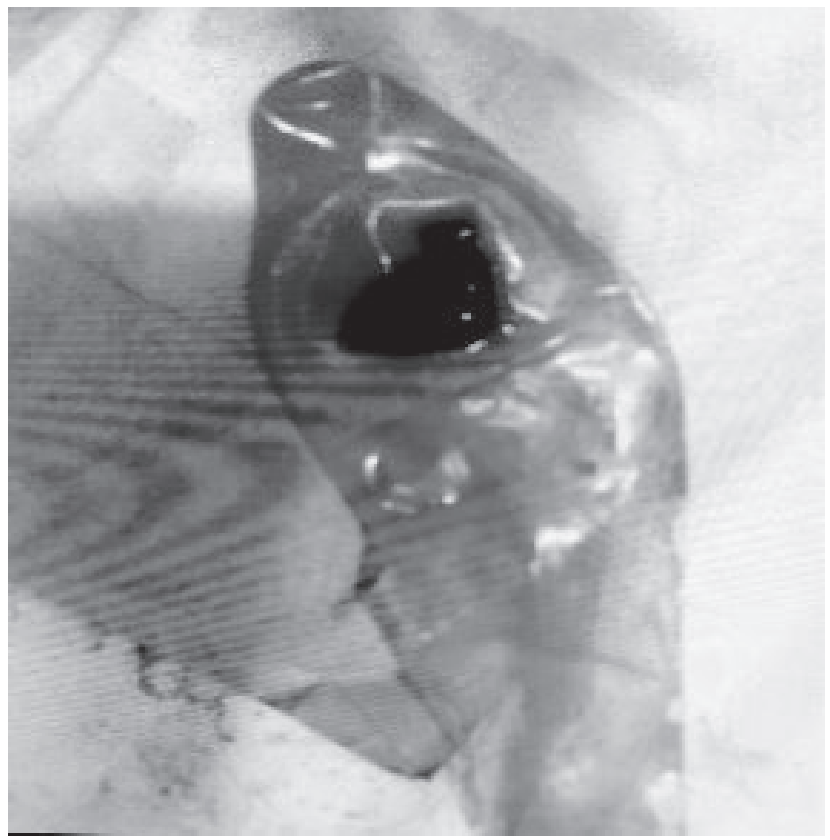

Fig.-6: Blood in semen in condom

\section{Result :}

Out of 30 cases studied, age of the patients was between 30 to 60 years. There were 13(43\%) patients of $(31-40) y r s, 08(27 \%)$ patients of $(41-50)$ yrs and $09(30 \%)$ patients of $(51-60)$ yrs Table-I shows age distribution of the patients.

\section{Table-I}

Digital rectal examination (DRE) finding of the study $\operatorname{cases}(n=30)$

\begin{tabular}{lcc}
\hline DRE finding & Number of patients & Percentage \\
\hline Enlarged prostate & 7 & $23 \%$ \\
Normal sized prostate & 23 & $77 \%$ \\
Any other finding & 0 & 0 \\
\hline
\end{tabular}

After DRE , among 30,23 patients found normal sized prostate and only 07 patients found enlarged prostate

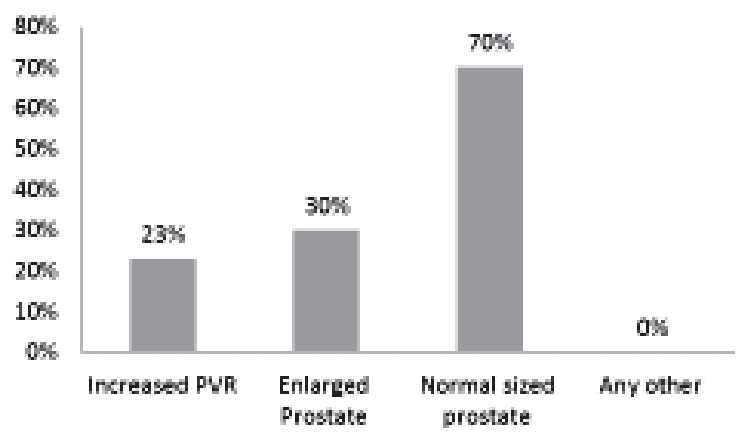

Fig.-7: Ultrasonogram (USG) finding of the patients

Fig VII showing USG findings and found 21 patients had normal size prostate and only 09 patients had enlarged prostate.07 patients found increased PVR.

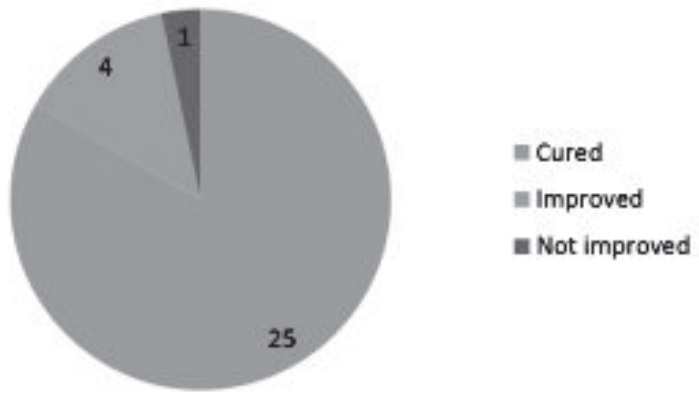

After per rectal laser therapy by optical probe there was improvement of symptoms found. 25 patients found cured and 04 patients found improved. Only 01 patients was not improved.

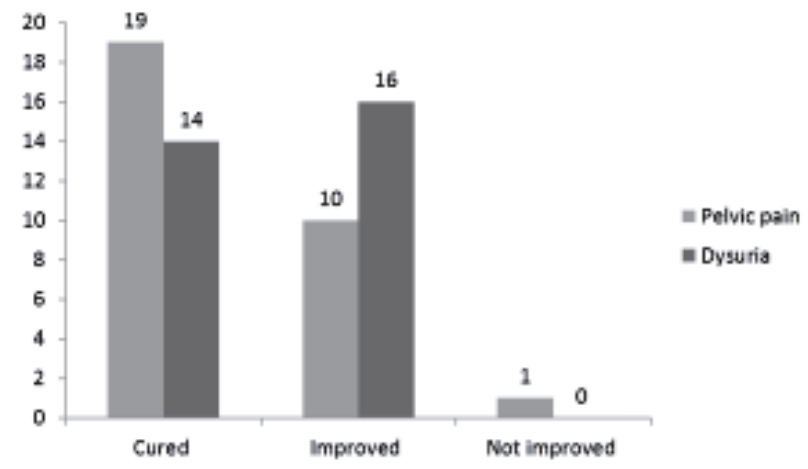

Fig.-9: Improvement of Other symptoms of CPPS after 
and 01 found not improved. 14 patients with dysuria found cured and 16 found improved.

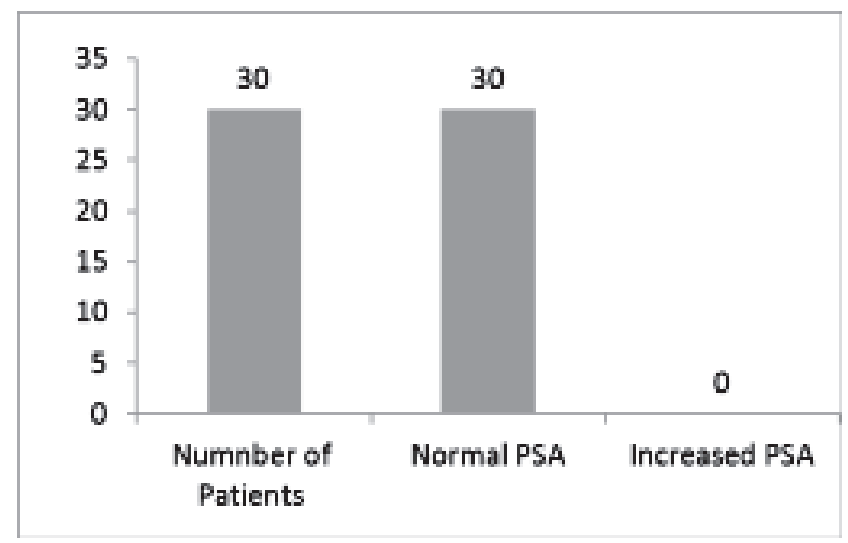

Fig.- 10: Serum PSA level

There was no significant findings of serum PSA .

\section{Pus cell in urine}

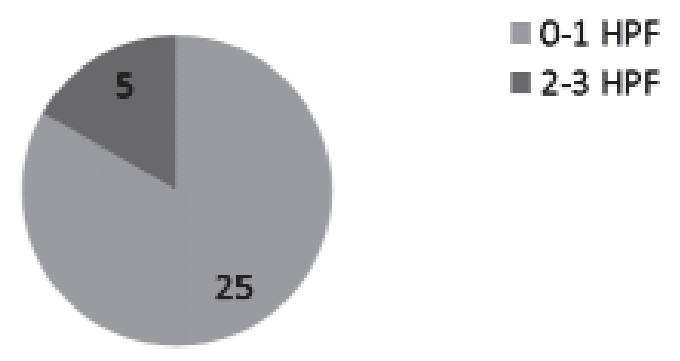

Fig.-11: Urine Analysis

25 patients found no pus cell in urine and only 05 patients found few pus cell in urine.

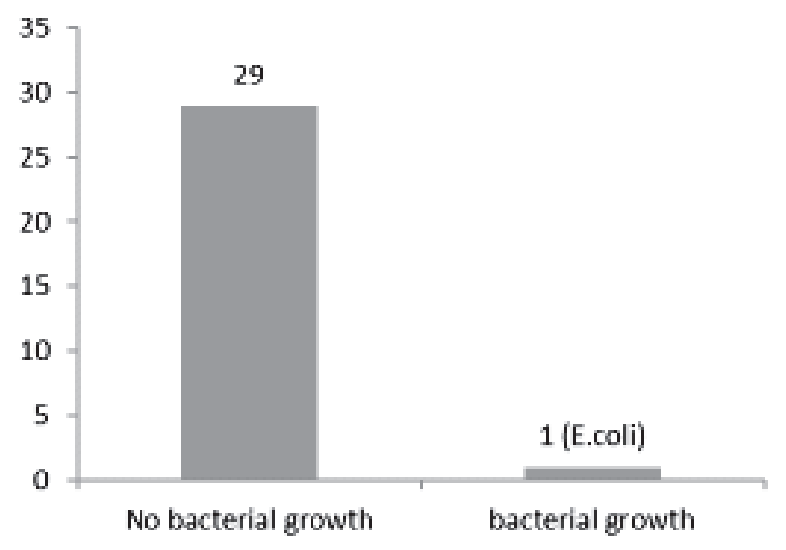

Fig-12: Bacterial growth in urine
29 patients found no bacterial growth in urine culture and onlt 01 found bacterial growth (E.coli) .

\section{Discussion:}

Haemospermia( haematospermia ) is a relatively frequent, distressing and frightening symptom in sexually active men. It is usually a benign self-limiting condition resolving within several weeks except for the few with underlying etiology including prostatitis and idiopathic . 5,7,8

Literature demonstrates, CPPS may occur from adolescence to old age. But this study shows youngest presentation at the age of 31 years. This may be due to the fact that our social infrastructure creates a feeling of shyness among the adolescent population to seek medical advice for such problems. The oldest patient in this series is 60 years. This could be due to lower life expectancy of people of this area..$^{9,10}$

Considering the symptomatology, pelvic pain and discomfort are the most common presentation which were present in $100 \%$ of the case studied. This correlates exactly with other different studies ${ }^{12,13,14}$. Other presentations include dysuria, haemospermia. The findings together with their frequency are exactly similar to certain other studies $11,12,13,14$.

Digital rectal examination (DRE) is necessary mainly to exclude LUTS due to prostatic enlargement- benign or malignant 14 . Prostate size was normal in $77 \%$ cases and mild enlargement in $23 \%$ cases.

Post void residual volume(PVR) and prostate size were found to be normal in most of the cases in this series. Literature also illustrates the same findings ${ }^{13,15}$.

A group of author found the patients of prostatitis /CPPS with haematospermia totally cured after application of MIL-Therapy . 16,17,18

After completion of laser therapy, patients were assessed for their improvement and they were followed up for a period of six months. Improvement of symptoms were graded on a subjective scale of cured, improved and not improved. As the scoring is subjective, there is an element of fallacy. It was found that, there was hardly any patient who did not improve. Majority were cured and rest had improvement with only one patient having no improvement of any symptom. Studies in Europe and America describes around $100 \%$ recovery after laser therapy $17,18,19$. The magic bullet is, there is no gross or significant complication following laser treatment both in literature and in this study ${ }^{18,19,20}$. 


\section{2(2) 2019}

Per Rectal MILT by optical probe and Haematospérmia with Chronic pelvic pain syndrome (CPPS) /Chronic Prostatitis

\section{Conclusion:}

Patients presenting with haematospermia should have a detailed medical history, physical examination including genital and digital rectal examination. Twothirds of the hematospermia patients were associated with evidence of prostatitis. We must carefully evaluate the association of prostatitis in hematospermia patients. Further, hematospermia patients with prostatitis revealed a relatively poor quality of life compared with those without prostatitis. To improve the quality of life of the patients, we must control the symptoms of CP/ CPPS in hematospermia patients with prostatitis. Persistant and recurrent haematospermia is best investigated by TRUS,CT,MRI, urethrocystoscopy and biopsies. Chronic pelvic pain syndrome (CPPS) is diagnosed by symptoms, signs, investigations and mainly through the methods of exclusion. This study has some limitation of small sample size, it should be better if sample size will more. The condition is poorly understood and difficult to treat. Further research is warranted to better understand the aetiological and pathogenesis of the condition and thereby improve the diagnosis and treatment of this common condition.

\section{References :}

1. (http://www.finetreatment.co.uk/naturalalternative/prostate-treatment.html)

2. A.M.Anamur Rashid Choudhury, Md. Waliul Islam, Tasmina Parveen, Feroze Quader, Md. Golam Mowla Choudhury . Effect of Magnetic Infrared -Laser therapy in Chronic Prostatitis/ Chronic Pelvic Pain Syndrome . J Uttara Adhunik Med Coll.2012 ; 2(2):100-104.

3. A. M. Anamur Rashid Choudhury, Md. Waliul Islam, Tasmina Parveen, Parveen Sultana .Per Rectal Magnetic Infra-Red Laser Therapy By Optical Probe And its effect on Pelvic pain , Dysuria and Haemospermia of the Patients of Chronic pelvic pain Syndrome. Bangladesh journal of Urology .2014; 17(1):42-46.

4. Rubinowicz DM,SolowayMS,Leif M, CivatosF. Hemospermia and expressed tumor in the urethra:an unusual presentation of ductal carcinoma of prostate. J Urol.2000;163:915

5. Munkelwitz R, Kransnokutsky $S$, LieJ,ShahSM,BayshtokJ,Khan SA. Current perspectives on haematospermia: a review .J Androl.1997;18:6-14.
6. Nickel JC. Pathogenesis of Chronic Protatitis. $\mathrm{Br}$ J Urol; 1990; 66: 47-49.

7. R.B. Alexander, K.J. Propert, A.J. Schaeffer, J.R. Landis, J.C. Nickel, M.P. O'Leary et al; Treating Men with Chronic Prostatitis/Chronic Pelvic Pain Syndrome,Annals of Internal Medicine; 2004, 141: 581-589.

8. Litwin MS. The National Institute of Health Chronic Prostatitis Symptom Index: development and validation of a new outcome measure. Chronic Prostatitis Collaborative Research Network. J Urol 1999; 162: 369-375.

9. Nickel JC, Nyberg LM, Hennenfent M. Research guidelines for chronic prostatitis: consensus report from the first National Institutes of Health International Prostatitis Collaborative Network. Urology 1999; 54: 229-233. .

10. American top urologists Wikipedia

11. Kaplan SA. Transurethral microwave hyperthermia and transurethral needle ablation. Prostate Cancer and Prostatic Diseases (2002) 5, 172-179.

12. Krieger JN, Nyberg L Jr, Nickel JC. NIH Consensus Definition and Classification of Prostatitis. JAMA 1999; 282: 236-237. Top of Form

13. file:///C:/DocumentsandSettings/user/Desktop/ CPPS/NationalGuideline/Chronic pelvic pain_htm

14. Meares EJ. Prostatitis. Med Clin North Am 1991; 75:405-407.

15. Berghuis JP. Psychological and Physiological factors involved in chronic idiopathic prostatitis. J Urol 1998; 159: 1422- 1433.

16. McNaughton W. Chronic prostatitis: a thorough search for etiologically involved microorganisms. Infection 1999;19:119-125.

17. Krieger WW, Jacob J, Ross SO. Detecting urethral and prostatic inflammation in patients with chronic prostatitid. Urology 2000; 55: 186-192.

18. Barbalias GA. Clinical and therapeutic guidelines for chronic prostatitis. Letter to the Editoe. Eur Urol 2000; 37: 116-117.

19. Dimitrakov J. Recent developments in diagnosis and therapy of the prostatitis syndromes. Curr Opinion Urol 2001; 11: 87-91. 
20. Choudhury AMAR, Islam MW, Parveen T, Sultana P.Per rectal Magnetic Infra -red Laser Therapy by optical probe and its effect on pelvic pain , dysuria and Haemospermia of patients of Chronic pelvic pain syndrome.Bangladesh J.Urol.2014;17(1):4246

21. Moon TD. Questionnare survey of urologists and primary care physicians' diagnostic and treatment practices for prostatitis. Urology 1997; 50: 543544.

22. Cho IR. Prostatic blood flow characteristics in the chronic prostatitis/ chronic pelvic pain syndrome. J Urol 2000; 163: 1130-1133.
23. Liu ZY,SunYH,XuCL, HouJG, GaoX, et al. Transurethral seminal vesiculoscopy in the diagnosis and treatment of persistant or recurrent hemospermia: a single -institution experience . Asian J Androl.2009;11:566-70.

24. $\mathrm{Ng} \mathrm{YH}$, Seeley JP, Smith G. Haematospermia as a presenting symptom: outcomes of investigation in 300 men. Surgeon 2013;11:35-8.

25.. Ahmad I, Krishna NS. Hemospermia. J Urol 2007;177: 1613-8. 3. Szlauer R, Jungwirth A. Haematospermia: diagnosis and treatment. Andrologia 2008;40:120-4. 